\title{
Reflex sensitization in the frog (Rana pipiens): Stimulation-independent influences
}

\author{
J. M. WHITNEY and R. LAHUE \\ University of Waterloo, Ontario N2L 3G1, Canada
}

\begin{abstract}
Daily stimulation of sites on the dorsum of leopard frogs can result in an enhancement of a leg-wiping reflex. This enhancement, while long lasting, is not specific, i.e., the eliciting of one reflex sensitizes reflexes of other limbs. Animals which receive handling each day but which are not stimulated also demonstrate an increased frequency of reflex elicitation though animals which don't receive daily handling do not. This, coupled with seasonal variations in responsivity suggested that nonspecific factors are largely responsible for enhancement of the reflex. The potential of this preparation as a simple system model for the study of learning mechanisms is questioned, and the need for detailed behavioral characterizations of such preparations prior to the undertaking of neurophysiological studies is emphasized.
\end{abstract}

Comparative investigations of learning mechanisms are making increasing use of "simple system" preparations in which naturally occurring or artificially induced restrictions in system complexity provide an enhanced possibility of elucidating fundamental mechanisms. Optimization of this strategy requires that systems be found which demonstrate modifiable behaviors as well as ease of electrophysiological and/or biochemical manipulation (Abraham, Palka, Peeke, \& Willows, 1971). Additionally, care must be taken to fully characterize the plasticity at the behavioral level lest spurious neuronal correlations be hypothesized (Lahue \& Corning, 1975). The present study helps to clarify an interesting phenomenon at the behavioral level and suggests that further studies of underlying mechanisms in the preparation need reconsideration.

Tactile stimulation of the dorsal skin of intact or spinal frogs occasionally elicits one of several wiping reflexes. The reflex elicited is specifically related to the site of stimulation, i.e., either the toes of the ipsilateral front or back leg or the heel of the back leg performs the wiping, dependent upon whether the anterior, major, or caudal zones, respectively, are stimulated. Franzisket (1963) reported that repetitive stimulation of a specific point resulted in an increased probability of eliciting the appropriate reflex. This enhancement was specific to the zone stimulated and persisted for over 20 days. Since the accessibility of the frog spinal cord to neurophysiological analysis has been well established, the possibility exists of exploring the mechanisms of this reflex sensitization. However, attempts at replication of the behavioral results with the readily available Rana pipiens (Franzisket used a European form, Rana esculenta have not been completely successful. Kimble and Ray (1964) found that slight variation in the site of

Requests for reprints should be sent to $R$. Lahue, Department of Psychology, University of Waterloo, Waterloo, Ontario N2L 3G1, Canada. stimulation was necessary in order to enhance the reflex above a $30 \%$ base rate. Repeated application of the stimulus to a very restricted locus resulted in a decrease in reflex elicitation. Corning and Lahue (1973) failed to find this habituation phenomenon. Rather, Franzisket's (1951, 1963) findings regarding enhancement were confirmed although no specificity could be demonstrated, i.e., stimulation of one zonal reflex resulted in the enhancement of the reflexes in other nonstimulated zones. Furthermore, simple handling of control frogs also increased the probability of reflex elicitation, and it was thought that the phenomenon might be an artifact of laboratory handling. While attempting to clarify previous results, the present experiment demonstrated the possibility of other influences upon reflex sensitivity.

\section{METHOD}

\section{Subjects}

Rana pipiens, 3 to 4 in. in length, were obtained from commercial suppliers at various times of the year and from different locations. For several days after arrival in the lab, the animals were maintained in a large stainless steel tank with continuously flowing water. This allowed the animals to recover from shipping stress before use. For experimentation, animals were randomly selected from the community tank and randomly assigned to both groups and treatments. The animals were then housed in plastic boxes ( $8 \times 10^{1 / 2} \times 6$ in. high) with glass tops. The bottom of the container was filled to a depth of $1 \frac{1 / 2}{2}$ in. with an artificial pond water composed of distilled water to which various salts had been added. This allowed for a standardized medium to be used all year long. The water in the cages was changed daily. All frogs were presented with meal worms daily but were not force fed. A 14:10 day-night cycle was maintained.

\section{Procedures}

A sketch made of the skin markings of each frog allowed repeated identification of stimulation sites since these were positioned relative to permanent markings. A point in a major zone was selected as a test stimulation site. Another point in the same zone, about $1 \mathrm{~cm}$ away, was used as a receptor specificity test site while one point in each of the other reflexogenous zones 
was designated as a reflex specificity test site. These specificity tests allowed an estimation of the extent to which reflex enhancement was related to restricted localization of stimulation in one zone as well as the degree to which the reflex activity of inactive legs was affected by training. For testing, frogs were removed from their home cages and were placed in a partially covered glass bowl under a red light to minimize responses to visual stimuli. On Days 1 and 11, all animals were treated in the same way. One hundred stimuli were presented to the test site with a 10 -sec ITI followed by the presentation of 10 stimuli to each of the specificity test sites at the same rate. On Days 2 through 10, 100 stimuli were applied to the test site in experimental frogs while an unstimulated handling control frog sat in a bowl nearby. Housing control animals were not treated during these 9 days.

All stimulation was accomplished with standardized bristles of the von Frey series, ranging in force from 3.84 to $4.74 \mathrm{~g} / \mathrm{mm}^{2}$. The probe selected for use on a given animal was that which, when applied perpendicularly to the surface of the back, just produced a slight dimpling of the skin followed by bending of the bristle. This allowed some equation of stimulus intensity over animals which may have differed slightly in size. The probe initially selected was used for all subsequent stimulation of a particular animal. To control stimulus intensity over trials and days, the same stimulation procedures (i.e., perpendicular application, skin dimpling, and bristle bending) were always observed. One experimenter ran the same animals over days with the treatment group identities concealed on Days 1 and 11 .

\section{RESULTS}

Two experiments were performed, in October and November. The first used frogs from Wisconsin while the second batch of frogs was from Manitoba. Each experiment consisted of eight animals per group, and since the results of the two experiments did not differ statistically, the data was combined. The three groups did not differ from one another in any way on the 1st day, and the average responsivity to the test stimulus was $11.3 \pm 4.8$ wipes per 100 stimuli. On Day 11 , the experimental and handling control groups exhibited a significant reflex enhancement over Day 1 with means of $33.2 \pm 10.0 \quad(\mathrm{~F}=9.11 ; \quad \mathrm{p}<.01)$ and $28.9 \pm 11.3$ $(\mathrm{F}=11.6 ; \mathrm{p}<.01)$ while not differing from one another $(F=1.04)$. On the other hand, no significant change in responsivity occurred in the housing control group which averaged $8.9 \pm 4.3$ wipes on Day 11. This was significantly lower than the responses of the other two groups $(F=17.55 ; p<.01)$. There was no demonstration of specificity in the experimental and handling control groups. Enhancement of one reflex was accompanied by an equivalent enhancement of all other reflexes. These results coincide rather closely with those of Corning and Lahue (1971) although the degree of reflex enhancement was somewhat larger in the earlier experiment. Since the fact that the earlier experiment was performed in winter might have been responsible for the differences, another two experiments using Manitoban and Wisconsin frogs (eight animals per group) were run, in January and February. The pattern of results was identical to that obtained in the fall experiments although the degree of reflex enhancement was closer to that of Corning and Lahue (1971). The means of the experimental and control groups for test responsivity on Day 11 were significantly higher than those of the first experiments $(67.6 \pm 18.6$ and $59.9 \pm 17.3$ wipes, respectively).

Preliminary to more detailed investigations of the reflex enhancement, recently purchased frogs were tested, as above, in May. No spontaneous reflex responsivity was observed in any of 24 animals, and 11 days of training did not alter responsivity. An additional 24 frogs from another source yielded the same result in late May. Several more experiments were run through August using freshly trapped frogs (i.e., they did not spend the winter in a laboratory) obtained variously from Mexico, Wisconsin, Manitoba, and Ontario. A variety of procedural modifications were attempted in order to produce the previously observed enhancement of reflex responsivity. For instance, the ionic composition of the water in which the animals were housed was altered, as were room temperature and lighting. Frequency of feeding was increased in some animals and decreased in others. Both heavier and light stimulation probes were used and regular stimulation was pursued for over 20 days. Nevertheless, a total of only nine wipes were elicited in all of these experiments, running from May through August and employing more than 150 frogs. This rate of responsivity is, of course, well below the Day 1 base rates observed in previous experiments. Thus, not only was no reflex enhancement observed, but the base rate of responsivity was essentially zero.

\section{DISCUSSION}

The finding here, and previously (Corning \& Lahue, 1971), that enhancement of one reflex in experimental animals resulted in increased responsivity for all wiping reflexes suggested that some nonspecific factor was involved in the enhancement. The fact that similar enhancement occurs in handling control animals, of course, indicates that the generalized responsivity increase is probably not causally related to the daily eliciting of one reflex but is rather an artifact of other procedures. The inclusion of a housing control group in the present study permits the conclusion that laboratory maintenance alone is insufficient to explain the increase in responsivity. While the degree to which central vs. peripheral factors may be involved in the behavioral plasticity demonstrated in handled animals could not be ascertained, the possibility does exist that some peripheral factor such as sensitization of skin receptors could account for the phenomenon. The finding that the degree of reflex enhancement obtained in winter exceeded that obtained in the fall suggested a possible interaction of some seasonal variable. The subsequent spring and summer experiments emphasized the importance of seasonal influences. Data concerning 
seasonal variations in Rana pipiens which might form the basis for the observed behavioral differences are lacking. However, investigations of other anurans have presented some possibilities. Ewert and Siefert (1974) have correlated neuronal behavior with seasonal variations in prey catching behavior in toads although possible mechanisms for this, such as metabolic or hormonal changes (see Porter, 1972), haven't been established. On the other hand, McClanahan (1972) reported changes in the ionic composition of the body fluids of burrowed (hibernating) toads as a function of the soil water potential. This, coupled perhaps, with physical stimulation attributed to the soil of the burrow and possible metabolic or hormonal cycles strongly suggests the possibility that skin receptor sensitivity may vary seasonally. The skin is, perhaps, quite insensitive following emergence in the spring and sensitivity doesn't seem to increase appreciably until fall. It is not known whether this eventual increase and the further enhancement later in winter are naturally occurring phenomena in the wild and are related to the onset of hibernation or are the result of studying animals which are deprived of burrowing and hibernation at the usual time of year. Further studies may clarify some of these questions, but it is clear that, at present, the potential that this preparation held for investigations of learning mechanisms has been overestimated. Such investigations are probably not worthwhile due to the complex interaction of factors likely to be involved in the response enhancement since they detract from the advantage of using a simple system preparation. Furthermore, the present work serves as a good example of the need to carefully delineate the characteristics of interesting behavioral changes before mechanisms of "learning" are sought. This is especially critical since many simple system preparations derive from wild (nonlaboratory) animal types, many of which are probably quite sensitive to the environmental alteration resulting from laboratory conditions.

\section{REFERENCES}

Abraham, F. D., Palka, J., Peeke, H. V. S., \& Willows, A. O. D. Model neural systems and strategies for the neurobiology of learning. Behavioral Biology, 1971, 7, 1-24.

Corning, W. C., \& Lahue, R. Reflex "training" in frogs. Psychonomic S̈cience, 1971, 23, 119-120.

Ewert, J. P., \& Siefert, G. Neuronal correlates of seasonal changes in contrast detection of prey catching behavior in toads (Bufo bufo L.). Vision Research, 1974, 14, 431-432.

Franzisket, L., Gewohnheitsbilduug und bedingte Reflexe bei Rückenmarkfröschen. Zeitschrift für vergleichende Phy siologie, 1951, 33, 142-178.

Franzisket, L. Characteristics of instinctive behavior and learning in reflex activity of the frog. Animal Behaviour, 1963, 11, 318-334.

Kimble, D. P., \& Ray, R. S. Reflex habituation and potentiation in Rana pipiens. Animal Behaviour, 1964, 13, 530-533.

Lahue, R \& Corning, W. C. Synthesis: A comparative look at vertebrates. In W. C. Corning, J. A. Dyal, and A. O. D. Willows (Eds.), Invertebrate learning (Vol. III). New York: Plenum Press, 1975.

McClanahan, J. Changes in body fluids of burrowed spadefoot toads as a function of soil water potential. Copeia, 1972, 1 , 209-215.

Porter, K. R. Herpetology. Philadelphia: W. B. Saunders, 1972.

(Received for publication December 6, 1974; revision accepted March 11, 1975.) 